\title{
Multiple-scale analysis and renormalization for preasymptotic scalar transport
}

\author{
A. Mazzino, ${ }^{1}$ S. Musacchio, ${ }^{2}$ and A. Vulpiani ${ }^{2,3}$ \\ ${ }^{1}$ INFM-Department of Physics, University of Genova, and INFN, Sezione di Genova, Via Dodecanneso 33, I-16146 Genova, Italy \\ ${ }^{2}$ Department of Physics and INFM (UdR and CSM), University "La Sapienza”, Piazzale Aldo Moro 2, I-00185 Roma, Italy \\ ${ }^{3}$ INFN, Sezione di Roma "La Sapienza", Piazzale Aldo Moro 2, I-00185 Roma, Italy
}

(Received 28 July 2004; revised manuscript received 25 October 2004; published 28 January 2005)

\begin{abstract}
Preasymptotic transport of a scalar quantity passively advected by a velocity field formed by a large-scale component superimposed on a small-scale fluctuation is investigated both analytically and by means of numerical simulations. Exploiting the multiple-scale expansion one arrives at a Fokker-Planck equation which describes the preasymptotic scalar dynamics. This equation is associated with a Langevin equation involving a multiplicative noise and an effective (compressible) drift. For the general case, no explicit expression for either the affective drift on the effective diffusivity (actually a tensorial field) can be obtained. We discuss an approximation under which an explicit expression for the diffusivity (and thus for the drift) can be obtained. Its expression permits us to highlight the important fact that the diffusivity explicitly depends on the large-scale advecting velocity. Finally, the robustness of the aforementioned approximation is checked numerically by means of direct numerical simulations.
\end{abstract}

DOI: 10.1103/PhysRevE.71.011113

PACS number(s): 05.40.-a, 05.60.Cd, 47.27.Qb, 47.27.Te

\section{INTRODUCTION}

Many problems, from biology to geophysics, include a variety of degrees of freedom with very different time scales [1]. As important examples of systems with multiple time scales we can mention protein folding and the climate. While the time scale for the vibration of covalent bonds is $O\left(10^{-15} \mathrm{~s}\right)$, the folding time for proteins may be of the order of seconds [2]. In an analogous way, climate dynamics involves processes with characteristic times ranging from days (atmosphere) to $10^{2}-10^{3} \mathrm{yr}$ (deep ocean and ice shields) [3].

Even modern supercomputers are not able to simulate all the relevant scales involved in such difficult problems. Consequently, scientists concerned with multiple-time-scale systems must develop suitable techniques for the treatment of the "slow dynamics" in terms of effective equations [1]. This is a very old problem: an early example of such techniques is the averaging method in mechanics. Starting from a system of $2 \mathrm{~N}$ ordinary differential equations written in the angleaction variables, where the angles $\left(\theta_{1}, \theta_{1}, \ldots, \theta_{N}\right)$ are "fast" and the actions $\left(I_{1}, I_{2}, \ldots, I_{N}\right)$ are "slow," the averaging method gives the leading order behavior of the actions by an effective equation for the averaged quantities $\left(\left\langle I_{1}\right\rangle,\left\langle I_{2}\right\rangle, \ldots,\left\langle I_{N}\right\rangle\right)$ obtained by averaging on the angles.

For the sake of self-consistency, we briefly recall the general problem. Let us limit ourselves to systems with only two times scales and denote by $\boldsymbol{x}$ and $\boldsymbol{y}$ the slow degrees of freedom and the fast ones, respectively. The time evolution is given by a set of ordinary differential equations:

$$
\begin{gathered}
\frac{d x}{d t}=f(x, y), \\
\frac{d y}{d t}=\frac{1}{\epsilon} g(x, y),
\end{gathered}
$$

where $\epsilon \ll 1$ is the ratio between the fast and slow characteristic time scales. The main goal is to approximate the motion of the slow variables $\boldsymbol{x}$ by an effective equation where the fast variables $\boldsymbol{y}$ do not appear.

Up to now, different methods have been proposed. Among the many, we can mention the Mori-Zwanzig formalism [4,5], invariant manifolds, averaging methods [6], conditional expectations [7], and Langevin equations [8,9].

Following the seminal works on Brownian motion $[10,11]$, it seems rather natural to mimic the dynamics of fast variables $\boldsymbol{y}$ through a white-in-time process, which amounts to describing the slow variables $\boldsymbol{x}$ in terms of a suitable Langevin equation. This approach is at the basis of the seminal paper of Hasselmann in climate modeling in terms of stochastic equations [8].

Under rather general conditions [12], one has the result that in the limit of small $\epsilon$ the slow dynamics is ruled by a Langevin equation with multiplicative noise:

$$
\frac{d \boldsymbol{x}}{d t}=\boldsymbol{f}_{\text {eff }}(\boldsymbol{x})+\boldsymbol{\sigma}(\boldsymbol{x}) \boldsymbol{\eta},
$$

where $\boldsymbol{\eta}$ is a white-noise vector, i.e., the components are Gaussian processes such that $\left\langle\eta_{i}(t)\right\rangle=0,\left\langle\eta_{i}(t) \eta_{j}\left(t^{\prime}\right)\right\rangle=\delta_{i j} \delta(t$ $\left.-t^{\prime}\right)$, and $\boldsymbol{\sigma}(\boldsymbol{x})$ is a tensorial field.

This class of problems attracts a great deal of attention in many field of physics, including, e.g., statistical physics. We just mention the celebrated renormalization group which can be seen as a technique to explicitly determine $\boldsymbol{f}_{\text {eff }}(\boldsymbol{x})$ and $\boldsymbol{\sigma}(\boldsymbol{x})$ in Hamiltonian systems [13]. There are rather general results $[14,15]$ that give explicit expression for the coefficients in Eq. (3) in terms of expectations over the fast process generated by Eq. (2) with slow $\boldsymbol{x}$ fixed. On the other hand there are technical difficulties in the practical use of such results, and therefore approximations based on physical ideas (such those in [4-8]) are required; for a recent review see [16]. Another interesting approach is to use the theoretical results in $[14,15]$ to built and test a numerical strategy for effective computation with Eq. (3) $[17,18]$. 
The aim of this paper is the investigation of a specific class of problems with many active, coupled degrees of freedom. To be more specific, we focus our attention on the large-scale transport of a scalar field $\theta(\boldsymbol{x}, t)$ advected by an incompressible velocity field consisting in the superposition of a large-scale, slowly varying, component and a smallscale, rapidly varying, fluctuation; namely,

$$
\partial_{t} \theta(\boldsymbol{x}, t)+\boldsymbol{v}(\boldsymbol{x}, t) \cdot \boldsymbol{\nabla} \theta(\boldsymbol{x}, t)=D_{0} \Delta \theta(\boldsymbol{x}, t)
$$

with

$$
\boldsymbol{v}(\boldsymbol{x}, t)=\boldsymbol{U}(\boldsymbol{x}, t)+\varepsilon \boldsymbol{u}(\boldsymbol{x}, t),
$$

where the typical length scale of $\boldsymbol{U}$ and $\boldsymbol{u}$ are $L$ and $\ell$, respectively, and $\ell / L \ll 1$. The parameter $\varepsilon$ controls the relative strength of the velocity components. It is worth recalling that Eq. (4) is nothing but the Fokker-Planck equation associated with the Langevin equation

$$
\frac{d \boldsymbol{x}(t)}{d t}=\boldsymbol{v}(\boldsymbol{x}, t)+\sqrt{2 D_{0}} \boldsymbol{\eta} .
$$

Our main aim here is to consider an effective large-scale transport equation for the large-scale scalar field $\theta_{L}$, varying on scales of the order of $L$, in which the dynamical effects of the smallest scales appear via a renormalized (enhanced) diffusivity. Such an equation reads [19]

$$
\partial_{t} \theta_{L}(\boldsymbol{x}, t)+\boldsymbol{U}(\boldsymbol{x}, t) \cdot \boldsymbol{\partial} \theta_{L}(\boldsymbol{x}, t)=\partial_{i}\left[D_{i j}(\boldsymbol{x}, t) \partial_{j} \theta_{L}(\boldsymbol{x}, t)\right]
$$

or, in the equivalent form,

$$
\partial_{t} \theta_{L}(\boldsymbol{x}, t)+\boldsymbol{\partial} \cdot\left[\boldsymbol{U}^{E}(\boldsymbol{x}, t) \theta_{L}(\boldsymbol{x}, t)\right]=\partial_{i} \partial_{j}\left[D_{i j}^{E}(\boldsymbol{x}, t) \theta_{L}(\boldsymbol{x}, t)\right],
$$

where

$$
\begin{gathered}
U_{i}^{E}(\boldsymbol{x}, t) \equiv\left[U_{i}(\boldsymbol{x}, t)+\partial_{j} D_{i j}(\boldsymbol{x}, t)\right], \\
D_{i j}^{E} \equiv \frac{D_{i j}+D_{j i}}{2} .
\end{gathered}
$$

We anticipate that $D_{i j}(\boldsymbol{x}, t)$ is in general neither symmetric nor defined as positive. Its symmetric part (which is also defined as positive) contributes to the diffusion process while both the symmetric and the antisymmetric parts enter, in general, in the effective advection velocity $\boldsymbol{U}^{E}$ which turns out to be compressible. As we will show, we have identified a sufficient condition which rules out the antisymmetric contribution of $D_{i j}(\boldsymbol{x}, t)$ in $\boldsymbol{U}^{E}(\boldsymbol{x}, t)$. In this case, $D_{i j}^{E}(\boldsymbol{x}, t)$ is the only relevant (in general unknown) field of the problem.

The Eulerian view for the large-scale dynamics given by Eq. (8) is equivalent to the Lagrangian description (written in the Itô formalism)

$$
\frac{d \boldsymbol{x}(t)}{d t}=\boldsymbol{U}^{E}(\boldsymbol{x}, t)+\sqrt{2 D_{i j}^{E}(\boldsymbol{x}, t)} \boldsymbol{\eta} .
$$

Unfortunately, although we know the equation for the preasymptotic dynamics of a scalar field, no explicit expression for $D_{i j}(\boldsymbol{x}, t)$ is available in general. We will discuss in the paper how to proceed perturbatively in $\varepsilon$ [the parameter defined in Eq. (5)] in order to obtain an approximate explicit expression for $D_{i j}(\boldsymbol{x}, t)$. Other than for applicative purposes, the advantage of this expression is that it permits us to highlight the important result that $D_{i j}(\boldsymbol{x}, t)$ explicitly depends on the large-scale advection $\boldsymbol{U}$. This is unlike the common way of thinking of an eddy-diffusivity contribution as the result of interactions involving only the small scales. Finally, we will show the results of direct numerical simulation in order to assess the robustness of the approximation and thus of the underlying physical mechanisms at the basis of the dependence of $D_{i j}(\boldsymbol{x}, t)$ on the large-scale velocity field.

In more detail, the paper is organized as follow. In Sec. II we will show how to derive Eq. (7) exploiting the multiplescale strategy (see, e.g., [19-23]). The latter is a renormalized perturbation method which requires one to have $\ell / L$ $\ll 1$. In general, the determination of the effective parameters can be performed only numerically (see, e.g., $[19,22])$. If, in addition to $\ell / L \ll 1$, we also assume $\varepsilon \ll 1$, an explicit expression for $D_{i j}(\boldsymbol{x}, t)$ can be derived. Some important conclusions can be drawn. Apart from the (trivial) case of shear flow, $D_{i j}(\boldsymbol{x}, t)$ cannot be constant; the components of the diffusivity tensor depend on the large-scale velocity as well as on the small scales. This latter point seems to be relevant for geophysical applications where such dependence on largescale flow is often not considered.

In Sec. III we will compute $D_{i j}(\boldsymbol{x}, t)$ perturbatively in $\varepsilon$. Only the leading, $O(\varepsilon)$, term of the series will be calculated analytically. This term is exact in some particular cases. Although also for the higher-order terms analytical expressions can be given, their complexity does not permit us to extract relevant information.

Numerical simulations performed on the exact Eq. (4) show that the approximate first-order solution is in very good agreement with numerical simulations also for $\varepsilon$ and $\ell / L$ not too small, say $0.2-0.4$. In addition we propose an empirical "recipe" to obtain a constant eddy diffusivity for preasymptotic transport. This is shown in Sec. IV.

In Sec. V we will discuss how, at least in principle, in the presence of velocity fields $\boldsymbol{u}(\boldsymbol{x}, t)$ containing contributions at many different scales, the multiple-scale approach can be iterated, in such a way that a renormalization group procedure naturally emerges with the result that an effective equation for asymptotic scales which involves an effective diffusivity $D^{E}$ can be obtained. Because of technical difficulties, the explicit detailed computation of the iteration procedure appears quite cumbersome. Nevertheless, for the dependence of $D^{E}$ on the velocity field, one can derive (and generalize) some results previously obtained in a phenomenological way.

Finally, Sec. VI is reserved for final conclusions and open problems.

\section{MULTIPLE-SCALE ANALYSIS}

Multiple-scale analysis applied to transport phenomena (see, e.g., Ref. [21]) constitutes a powerful tool to extract the equations ruling the large-scale dynamics from first principles, i.e., the equations describing the entire set of spatial and temporal degrees of freedom.

From a general point of view, the large-scale equations involve renormalized parameters which can usually be deter- 
mined by solving an auxiliary differential problem that requires the knowledge of the fully resolved fields. This is, for instance, the case analyzed in Ref. [23] where it is shown that the large-scale dynamics of a scalar field, in the presence of scale separation with respect to the (small-scale) advecting velocity field, is governed by an effective equation that is always diffusive. The diffusion coefficient (actually a tensor) turns out to be larger than the bare (molecular) diffusion coefficient: unsolved turbulent motion enhances the largescale transport (see Ref. [20]).

The latter result has been generalized in Ref. [19] where the preasymptotic passive scalar dynamics has been analyzed. There, the assumption of dealing with a small-scale advecting velocity field has been relaxed and the possible dependence of velocity on scales comparable with those of the scalar has been taken into account. As a result, we will show here that the large-scale (preasymptotic) equation does not have a Fokker-Planck structure although it involves a renormalized diffusivity (actually a tensorial field). The latter is varying on scales comparable with those of the large-scale components of the advecting velocity. As a consequence, no Lagrangian description is associated with such a Eulerian equation.

\section{A. Preasymptotic dynamics of a passive scalar: Heuristic considerations}

The starting point of our analysis is the equation ruling the evolution of a passive scalar field $\theta(\boldsymbol{x}, t)$ in an incompressible velocity field $\boldsymbol{v}$ :

$$
\partial_{t} \theta(\boldsymbol{x}, t)+\boldsymbol{v} \cdot \boldsymbol{\nabla} \theta(\boldsymbol{x}, t)=D_{0} \Delta \theta(\boldsymbol{x}, t) .
$$

If one is interested in studying the scalar dynamics in the deep infrared limit (i.e., very large scales) the proper choice for $\boldsymbol{v}$ is as in Ref. [20]: a small-scale field varying on scales well separated from those at which the scalar dynamics is observed.

More frequently, in real applications (e.g., in geophysics) one could be interested in studying the scalar dynamics on large scales where the advecting velocity is, however, still relevant (i.e., at such wave numbers the velocity is appreciably nonzero). Following Ref. [19], the simplest way to treat a similar situation is to decompose $\boldsymbol{v}$ as the sum of $\boldsymbol{u}(\boldsymbol{x}, t)$ and $\boldsymbol{U}(\boldsymbol{x}, t)$. The former is assumed to vary on what we call "small scales" [i.e., wave numbers of $O(1)$ ] while the latter evolves on "large scales" having wave numbers of $O(\epsilon)$, the same at which we aim at investigating the scalar dynamics.

Naive arguments would suggest a simple (wrong) conclusion: $\boldsymbol{U}(\boldsymbol{x}, t)$ gives the advection contribution in the largescale equation for $\theta$ while the renormalized diffusion coefficient emerges from small-scale interactions between $\theta$ and $\boldsymbol{u}$. A detailed analysis actually shows that this conclusion is wrong: the large-scale velocity $\boldsymbol{U}(\boldsymbol{x}, t)$ is not responsible for only the large-scale advection; it also enters in the renormalized diffusivity.

Before proceeding with a formal derivation where this effect clearly emerges, let us give a heuristic argument in favor of such a mechanism.
Suppose we have a large-scale initial condition for $\theta$ at time $t=0$ behaving on wave numbers of $O(\epsilon)$ and, moreover, $\boldsymbol{v}=\boldsymbol{u}$ (i.e., the case discussed in Ref. [20]). Due to the advection term $\boldsymbol{u} \cdot \boldsymbol{\nabla}$ in Eq. (4), scalar components with wave numbers of $O(1+\epsilon)$ are excited at larger times. The latter scalar components can interact, again due to the action of $\boldsymbol{u} \cdot \boldsymbol{\nabla}$, with those of $\boldsymbol{u}$ to generate, at successive times, large-scale components of $\theta$. This is the basic mechanism giving rise to the renormalization of the bare diffusion coefficient via interaction involving small scales.

Let us now repeat the argument in the presence of $\boldsymbol{U}$ which varies on the scale of wave numbers of $O(\epsilon)$. The interactions we have described above continue to work with the main difference that new contributions to the wave numbers of $O(1+\epsilon)$ now come from interactions of $O(\epsilon)$ modes of $\boldsymbol{U}$ and $O(1)$ modes of $\theta$. The latter modes being involved in the renormalization process, one can conclude that $\boldsymbol{U}$ plays a role in such renormalization. Whether or not this is really the case requires a formal analysis, which is the subject of the next section.

\section{B. Formal analysis for the preasymptotic scalar transport}

Following Ref. [19], let us decompose $\boldsymbol{v}$ as $\boldsymbol{v}(\boldsymbol{x}, t)$ $\equiv \boldsymbol{U}(\boldsymbol{x}, t)+\boldsymbol{u}(\boldsymbol{x}, t)$ where $\boldsymbol{U}(\boldsymbol{x}, t)$ and $\boldsymbol{u}(\boldsymbol{x}, t)$ are assumed to be periodic in boxes of sides $O\left(\epsilon^{-1}\right)$ and $O(1)$, respectively. (The technique we are going to describe can be extended with some modifications to handle the case of a random, homogeneous, and stationary velocity field.)

Our focus is on the large-scale dynamics of the field $\theta(\boldsymbol{x}, t)$ on spatial scales of $O\left(\epsilon^{-1}\right)$. In the spirit of multiplescale analysis, we introduce a set of slow variables $\boldsymbol{X}=\boldsymbol{\epsilon} \boldsymbol{x}$, $T=\epsilon^{2} t$, and $\tau=\epsilon t$ in addition to the fast variables $(\boldsymbol{x}, t)$. The scaling of the times $T$ and $\tau$ is suggested by physical reasons: we are searching for diffusive behavior on large time scales of $O\left(\epsilon^{-2}\right)$ taking into account the effects played by the advection contribution occurring on time scales of $O\left(\epsilon^{-1}\right)$.

The prescription of the technique is to treat the variables as independent. It then follows that

$$
\begin{gathered}
\partial_{i} \mapsto \partial_{i}+\epsilon \nabla_{i}, \quad \partial_{t} \mapsto \partial_{t}+\epsilon \partial_{\tau}+\epsilon^{2} \partial_{T}, \\
\boldsymbol{u} \mapsto \boldsymbol{u}(\boldsymbol{x}, t), \quad \boldsymbol{U} \mapsto \boldsymbol{U}(\boldsymbol{X}, T),
\end{gathered}
$$

where $\partial$ and $\nabla$ denote the derivatives with respect to fast and slow space variables, respectively. The solution is sought as a perturbative series

$$
\theta(\boldsymbol{x}, t ; \boldsymbol{X}, T ; \tau)=\theta^{(0)}+\epsilon \theta^{(1)}+\epsilon^{2} \theta^{(2)}+\cdots,
$$

where the functions $\theta^{(n)}$ depend, a priori, on both fast and slow variables. By inserting Eqs. (15) and (13) into Eq. (4) and equating terms having equal powers in $\epsilon$, we obtain a hierarchy of equations in which both fast and slow variables appear. The solutions of interest to us are those having the same periodicities as the velocity field $\boldsymbol{u}(\boldsymbol{x}, t)$.

By averaging such equations over the small-scale periodicity (here denoted by $\langle\cdot\rangle$ ), a set of equations involving only the large-scale fields (i.e., depending on $\boldsymbol{X}, T$, and $\tau$ ) are easily obtained. Obviously, such equations must be solved 
recursively, because of the fact that solutions of a given order appear as coefficients in the equations at the higher orders. Let us show in detail this point.

It is not difficult to verify that the equations at order $\epsilon$ and $\epsilon^{2} \operatorname{read}[19]$

$$
\begin{gathered}
O(\epsilon): \partial_{t} \theta^{(1)}+(\boldsymbol{v} \cdot \boldsymbol{\partial}) \theta^{(1)}-D_{0} \partial^{2} \theta^{(1)}=-(\boldsymbol{v} \cdot \boldsymbol{\nabla}) \theta^{(0)}-\partial_{\tau} \theta^{(0)} \\
\begin{aligned}
& O\left(\epsilon^{2}\right): \partial_{t} \theta^{(2)}+(\boldsymbol{v} \cdot \boldsymbol{\partial}) \theta^{(2)}-D_{0} \partial^{2} \theta^{(2)} \\
&=- \partial_{T} \theta^{(0)}-(\boldsymbol{v} \cdot \boldsymbol{\nabla}) \theta^{(1)}+D_{0} \nabla^{2} \theta^{(0)} \\
&+2 D_{0}(\boldsymbol{\partial} \cdot \boldsymbol{\nabla}) \theta^{(1)}-\partial_{\tau} \theta^{(1)} .
\end{aligned}
\end{gathered}
$$

The linearity of Eq. (17) permits us to search for a solution in the form

$\theta^{(1)}(\boldsymbol{x}, t ; \boldsymbol{X}, T ; \tau)=\left\langle\theta^{(1)}\right\rangle(\boldsymbol{X}, T ; \tau)+\boldsymbol{\chi}(\boldsymbol{x}, t ; \boldsymbol{X}, T) \cdot \boldsymbol{\nabla} \theta^{(0)}(\boldsymbol{X}, T ; \tau)$,

where $\theta^{(0)}$ depends only on the large-scale variables as in Ref. [20]. Plugging Eq. (18) into the solvability condition for Eq. (17), one obtains the equation

$$
\partial_{T} \theta^{(0)}+(\boldsymbol{U} \cdot \boldsymbol{\nabla})\left\langle\theta^{(1)}\right\rangle+\partial_{\tau}\left\langle\theta^{(1)}\right\rangle=\nabla_{i}\left(D_{i j} \nabla_{j} \theta^{(0)}\right),
$$

where

$$
D_{i j}(\boldsymbol{X}, T)=\delta_{i j} D_{0}-\left\langle u_{i} \chi_{j}\right\rangle
$$

is a second-order tensorial field and $\boldsymbol{\chi}(\boldsymbol{x}, t ; \boldsymbol{X}, T)$ has a vanishing average over the periodicities and satisfies the following equation:

$$
\partial_{t} \chi_{j}+[(\boldsymbol{u}+\boldsymbol{U}) \cdot \boldsymbol{\partial}] \chi_{j}-D_{0} \partial^{2} \chi_{j}=-u_{j} .
$$

Note that, when $\boldsymbol{U}$ is not a pure mean flow but depends on $\boldsymbol{X}$ and $T$, Eq. (21) must be solved for each value of $\boldsymbol{X}$ (and eventually $T$ ). (16),

From Eq. (19) and from the solvability condition of Eq.

$$
\partial_{\tau}\left\langle\theta^{(0)}\right\rangle+(\boldsymbol{U} \cdot \boldsymbol{\nabla})\left\langle\theta^{(0)}\right\rangle=0,
$$

one obtains the equation for the large-scale field $\theta_{L}$ defined as $\theta_{L} \equiv\left\langle\theta^{(0)}\right\rangle+\epsilon\left\langle\theta^{(1)}\right\rangle$ :

$$
\partial_{t} \theta_{L}+(\boldsymbol{U} \cdot \boldsymbol{\partial}) \theta_{L}=\partial_{i}\left(D_{i j} \partial_{j} \theta_{L}\right),
$$

where the usual variables $\boldsymbol{x}, t$ are used.

The important point to note is that $D_{i j}$ is in general neither symmetric nor defined positive. On the contrary, it is easy to show [19] that $D_{i j}^{E} \equiv\left(D_{i j}+D_{j i}\right) / 2$ is (obviously) symmetric and defined positive. Its expression can immediately be obtained from Eq. (21) in term of only the auxiliary field:

$$
D_{i j}^{E}=D_{0}\left\langle\partial_{p} \chi_{i} \partial_{p} \chi_{j}\right\rangle .
$$

In terms of $D_{i j}^{E}$ and $D_{i j}^{A} \equiv\left(D_{i j}-D_{j i}\right) / 2$, the preasymptotic equation (23) takes the form

$$
\partial_{t} \theta_{L}+\boldsymbol{\partial} \cdot\left(\boldsymbol{U}^{E} \theta_{L}\right)=\partial_{i} \partial_{j}\left(D_{i j}^{E} \theta_{L}\right),
$$

where

$$
U_{i}^{E}(\boldsymbol{x}, t) \equiv\left[U_{i}(\boldsymbol{x}, t)+\partial_{j} D_{i j}^{E}(\boldsymbol{x}, t)+\partial_{j} D_{i j}^{A}(\boldsymbol{x}, t)\right]
$$

is an effective compressible advecting velocity [24]. Advection by compressible velocities have been investigated, e.g., in Refs. [25,26].

\section{Formal analysis for the asymptotic scalar transport}

Our aim is now to investigate transport on scales much larger than the typical length of the field $\boldsymbol{U}$, i.e., on scales $\mathcal{L} \gg L$.

Homogenization leads to a purely diffusive dynamics which involves a set of new slow variables $\mathcal{X}=\epsilon^{\prime} \boldsymbol{X}$ and $\mathcal{T}$ $=\epsilon^{\prime 2} T$ describing the large-scale field $\theta_{\mathcal{L}}=\left\langle\theta_{L}\right\rangle$. Averages are now over the cell of size $L$ :

$$
\partial_{\mathcal{T}} \theta_{\mathcal{L}}=D_{i j}^{\mathcal{L}} \nabla_{i} \nabla_{j} \theta_{\mathcal{L}} .
$$

There are two different ways to arrive at the large-scale equation (27). The first way is to apply the homogenization technique from Eq. (23) while the second possibility is to start directly from Eq. (4). Let us consider the first option. In this case, the asymptotic eddy-diffusivity tensor $D^{\mathcal{L}}$ will then result from the combined effects of the advection given by the large-scale flow $\boldsymbol{U}(\boldsymbol{X}, T)$ and the diffusion at scale $\ell$ which also depend on space and time through $D_{i j}(X, T)$,

$$
\begin{aligned}
D_{i j}^{\mathcal{L}}= & -\frac{\left\langle U_{i} \chi_{j}\right\rangle+\left\langle U_{j} \chi_{i}\right\rangle}{2}+\frac{\left\langle D_{i k} \partial_{k} \chi_{j}\right\rangle+\left\langle D_{j k} \partial_{k} \chi_{i}\right\rangle}{2} \\
& +\frac{\left\langle D_{i j}\right\rangle+\left\langle D_{j i}\right\rangle}{2},
\end{aligned}
$$

where the vector field $\boldsymbol{\chi}$ is here a solution of the auxiliary equation

$$
\partial_{t} \chi_{k}+(\boldsymbol{U} \cdot \boldsymbol{\partial}) \chi_{k}-\partial\left(D_{i j} \partial_{j} \chi_{k}\right)=-U_{k}+\partial_{i} D_{i k} .
$$

If one follows the second way to obtain the large-scale equation (27), the (exact) value of the eddy-diffusivity tensor $D^{\mathcal{L}, e x}$ depends on both the molecular diffusivity and the advection by the total velocity field $\boldsymbol{v}=\boldsymbol{U}+\boldsymbol{u}$ :

$$
D_{i j}^{\mathcal{L}, e x}=\delta_{i j} D_{0}-\frac{\left\langle v_{i} \chi_{j}\right\rangle+\left\langle v_{j} \chi_{i}\right\rangle}{2} .
$$

Here, the auxiliary field $\boldsymbol{\chi}$ is the solution of the following equation:

$$
\partial_{t} \boldsymbol{\chi}+(\boldsymbol{v} \cdot \boldsymbol{\partial}) \boldsymbol{\chi}-D_{0} \partial^{2} \boldsymbol{\chi}=-\boldsymbol{v} .
$$

The latter procedure gives the exact value of the eddydiffusivity tensor $D^{\mathcal{L}, e x}$, but requires the detailed knowledge of the velocity field at both large and small scales. On the other hand, the expression obtained from Eq. (28) (which, in general, does not coincide with $D^{\mathcal{L}, e x}$ ) is based on only the large-scale velocity $\boldsymbol{U}$, and the effects of the small-scale flow are included in the eddy diffusivity $D_{i j}(\boldsymbol{X}, T)$.

A clear indication that $D^{\mathcal{L}, e x} \neq D^{\mathcal{L}}$ can be obtained by noting that the eddy-diffusivity tensor $D_{i j}$ does not depend on the relative position (i.e., possible spatial shifts) between the two fields $\boldsymbol{U}$ and $\boldsymbol{u}$. This is an obvious consequence of scale separation which washes out all detailed differences between 
the two fields. Therefore, the effects of relative shifts between $\boldsymbol{U}$ and $\boldsymbol{u}$ which are taken into account in the exact eddy-diffusivity tensor $D^{\mathcal{L}, e x}$ are missed by the approximate expression for $D^{\mathcal{L}}$. A comparison between the expressions for the asymptotic diffusivities obtained by following the two different homogenization procedures allows one to quantify the error of the approximate strategy.

It is worth mentioning a particular case in which both procedures lead to the same results. This is the case when the velocity field $\boldsymbol{v}$ is given by the sum of two parallel steady shears,

$$
\boldsymbol{v}(\boldsymbol{x} ; \boldsymbol{X})=\boldsymbol{u}(\boldsymbol{x})+\boldsymbol{U}(\boldsymbol{X}),
$$

with

$$
\boldsymbol{u}(\boldsymbol{x})=(u(y), 0), \quad \boldsymbol{U}(\boldsymbol{X})=(U(Y), 0),
$$

where $U$ and $u$ vary on scales of the order of $L$ and $\ell$, respectively.

A first homogenization on the small scales $\ell$ leads to an eddy diffusivity (we use the equivalent notations $D_{x x} \equiv D_{11}$ and $\left.D_{y y} \equiv D_{11}\right)$

$$
D_{x x}=D_{0}+\frac{1}{2} \int \frac{|\hat{u}|^{2} d k}{D_{0} k^{2}}, \quad D_{y y}=D_{0}, \quad D_{i j}=0 \quad \forall i \neq j .
$$

One can now repeat the same homogenization procedure at large scales $L$, obtaining

$$
D_{x x}^{\mathcal{L}}=D_{x x}+\frac{1}{2} \int \frac{|\hat{U}|^{2} d k}{D_{y y} k^{2}}=D_{0}+\frac{1}{2} \int \frac{|\hat{u}|^{2} d k}{D_{0} k^{2}}+\frac{1}{2} \int \frac{|\hat{U}|^{2} d k}{D_{0} k^{2}},
$$

which coincides with the exact coefficient obtained from the homogenization carried out from Eq. (4) which involves the total velocity field $\boldsymbol{v}=\boldsymbol{U}+\boldsymbol{u}$ :

$$
D_{x x}^{\mathcal{L}, e x}=D_{0}+\frac{1}{2} \int \frac{\left(|\hat{U}|^{2}+|\hat{u}|^{2}\right) d k}{D_{0} k^{2}} .
$$

\section{AN APPROXIMATE EXPRESSION FOR THE EDDY-DIFFUSIVITY FIELD}

In the previous section we have shown how to reduce the computation of the eddy-diffusivity tensor $D_{i j}(x, t)$ to the solution of an auxiliary equation. It is, however, worth noting that the parametric dependence on the large-scale variables $\boldsymbol{X}, T$ in the auxiliary field $\boldsymbol{\chi}(\boldsymbol{x}, t ; \boldsymbol{X}, T)$ in Eq. (21) imposes a rather severe limit to the practical use of Eq. (23). If the large-scale velocity $\boldsymbol{U}$ depends on space and time, one has indeed to solve an auxiliary equation in $(2 d+1)$ dimensions.

Therefore, except for very few cases in which one can obtain an analytic solution for $\boldsymbol{\chi}(\boldsymbol{x}, t ; \boldsymbol{X}, T)$, e.g., in the case of orthogonal shears (see Sec. III B), Eq. (20) does not provide a practical tool for evaluating the eddy diffusivity of generic flows. The computational cost required for the solution of the auxiliary equation can indeed be heavier than that required for the solution of the complete equation.
In the following we will show how the presence of an intense large-scale flow permits one to overcome this limit. Indeed, if the strength of the large-scale flow $\boldsymbol{U}$ is much larger than that of the small-scale velocity field $\boldsymbol{u}$, one can seek the solution of the auxiliary equation as a perturbative series in the small parameter $\varepsilon=u / U$ :

$$
\boldsymbol{\chi}(\boldsymbol{x}, t ; \boldsymbol{X}, T)=\boldsymbol{\chi}^{(0)}+\varepsilon \boldsymbol{\chi}^{(1)}+\varepsilon^{2} \boldsymbol{\chi}^{(2)}+\cdots,
$$

where the functions $\boldsymbol{\chi}^{(n)}$ depend on both fast and slow variables. By inserting Eq. (37) into Eq. (21) and equating terms having equal powers in $\varepsilon$, we obtain a hierarchy of equations:

$$
\begin{gathered}
\partial_{t} \boldsymbol{\chi}^{(0)}+(\boldsymbol{U} \cdot \boldsymbol{\partial}) \boldsymbol{\chi}^{(0)}-D_{0} \partial^{2} \boldsymbol{\chi}^{(0)}=0, \\
\partial_{t} \boldsymbol{\chi}^{(1)}+(\boldsymbol{U} \cdot \boldsymbol{\partial}) \boldsymbol{\chi}^{(1)}-D_{0} \partial^{2} \boldsymbol{\chi}^{(1)}=-\boldsymbol{u},
\end{gathered}
$$

$$
\partial_{t} \boldsymbol{\chi}^{(n)}+(\boldsymbol{U} \cdot \boldsymbol{\partial}) \boldsymbol{\chi}^{(n)}-D_{0} \partial^{2} \boldsymbol{\chi}^{(n)}=-(\boldsymbol{u} \cdot \boldsymbol{\partial}) \boldsymbol{\chi}^{(n-1)} .
$$

The zeroth-order equation has the trivial solution $\boldsymbol{\chi}^{(0)}=\boldsymbol{\chi}^{(0)}(\boldsymbol{X}, T)$, which clearly does not contribute [see Eq. (20)] to $D_{i j}(\boldsymbol{X}, T)$, while the higher-order equations can be easily solved in Fourier space. At first order in $\varepsilon$ the solution reads

$$
\hat{\boldsymbol{\chi}}^{(1)}(\boldsymbol{k}, \omega ; \boldsymbol{X}, T)=\frac{-\hat{\boldsymbol{u}}(\boldsymbol{k}, \omega)}{i(\omega+\boldsymbol{U} \cdot \boldsymbol{k})+k^{2} D_{0}},
$$

which, exploiting Eq. (20), leads to the following expression:

$$
\begin{aligned}
D_{i j}(\boldsymbol{X}, T)= & D_{0} \delta_{i j}+\int d \boldsymbol{q} d \omega\left\{\frac{\operatorname{Re}\left[\hat{u}_{i}(-\boldsymbol{q},-\omega) \hat{u}_{j}(\boldsymbol{q}, \omega)\right] q^{2} D_{0}}{(\omega+\boldsymbol{U} \cdot \boldsymbol{q})^{2}+q^{4} D_{0}^{2}}\right. \\
& \left.+\frac{\operatorname{Im}\left[\hat{u}_{i}(-\boldsymbol{q},-\omega) \hat{u}_{j}(\boldsymbol{q}, \omega)\right](\omega+\boldsymbol{U} \cdot \boldsymbol{q})}{(\omega+\boldsymbol{U} \cdot \boldsymbol{q})^{2}+q^{4} D_{0}^{2}}\right\}+O\left(\varepsilon^{3}\right) .
\end{aligned}
$$

Equation (42) permits one to highlight some important points. The eddy diffusivity is not simply determined by the small-scale flow: it actually has an explicit dependence on the large-scale velocity components. A rough estimation of the eddy diffusivity based on the sole small-scale field can lead to completely wrong results when a large-scale flow is present. Moreover, the variation in space and time of the velocity field $\boldsymbol{U}(\boldsymbol{X}, T)$ induces an implicit dependence on the slow variables $\boldsymbol{X}, T$ in the eddy diffusivity, which thus becomes a tensorial field. We stress the fact that such a dependence on $X, T$ is not a consequence of the approximation (42), the same property holds if one use the exact $\chi$.

The physical origin of this effect is the strong sweeping caused by the large-scale velocity field, which changes the effective correlation times of the small-scale flow. Therefore, the frequencies $\omega$ that appear in Eq. (41) experiences a Doppler shift corresponding to the inverse of the sweeping time $\boldsymbol{U} \cdot \boldsymbol{k}$. Only when the temporal variation of the small-scale flow is much faster than the large-scale sweeping, i.e., when the power spectrum of the small-scale flow is peaked at very high frequencies $\omega \gg \boldsymbol{U} \cdot \boldsymbol{k}$, one obtains a constant tensor which does not depend on $\boldsymbol{U}$ : 


$$
\begin{aligned}
D_{i j}(\boldsymbol{X}, T)= & D_{0} \delta_{i j}+\int\left\{\frac{\operatorname{Re}\left[\hat{u}_{i}(-\boldsymbol{q},-\omega) \hat{u}_{j}(\boldsymbol{q}, \omega)\right] q^{2} D_{0}}{\omega^{2}+q^{4} D_{0}^{2}}\right. \\
& \left.+\frac{\operatorname{Im}\left[\hat{u}_{i}(-\boldsymbol{q},-\omega) \hat{u}_{j}(\boldsymbol{q}, \omega)\right] \omega}{\omega^{2}+q^{4} D_{0}^{2}}\right\} d \boldsymbol{q} d \omega+O\left(\varepsilon^{3}\right) .
\end{aligned}
$$

As we have already shown in Sec. II B, both the symmetric and the antisymmetric parts of $D_{i j}$ contribute to the effective advecting velocity

$$
U_{i}^{E}(\boldsymbol{x}, t) \equiv U_{i}(\boldsymbol{x}, t)+\partial_{j} D_{i j}^{E}(\boldsymbol{x}, t)+\partial_{j} D_{i j}^{A}(\boldsymbol{x}, t) .
$$

Exploiting the explicit expression for $D_{i j}$ it is easy to derive a sufficient condition under which $D_{i j}^{A}$ is identically zero [and thus $\left.\partial_{j} D_{i j}^{A}(\boldsymbol{x}, t)=0\right]$. If this condition is satisfied, then only $D_{i j}^{E}$ is relevant for the dynamics at preasymptotic scales. This seems interesting for applications in view of the fact that, in three dimensions, only six, rather than nine, fields (the components of $D_{i j}^{E}$ ) must be taken into account.

Formally, the analytic result obtained for the eddy diffusivity is valid only in the limits $\ell / L \ll 1$ and $u / U \ll 1$. Therefore we must expect some discrepancies between the actual results for $\ell / L \sim 1$ and $u / U \sim 1$ and those obtained exploiting the multiple-scale method. Actually, we will show in Sec. IV that good results are obtained even if the ratio between the characteristic scales of the flows, $\epsilon=\ell / L$, and amplitudes, $\varepsilon=u / U$, are not too small.

\section{A. A sufficient condition for the effective advecting velocity}

As a starting point let us rewrite $\hat{u}_{i}$ in term of its real and imaginary parts, $\hat{u}_{i}=\hat{u}_{i}^{\mathrm{R}}+\sqrt{-1} \hat{u}_{i}^{\mathrm{I}}$, and plug it into Eq. (42) which takes the form

$$
\begin{aligned}
D_{i j}(\boldsymbol{X}, T)= & D_{0} \delta_{i j}+\int\left\{\frac{q^{2} D_{0}}{(\omega+\boldsymbol{U} \cdot \boldsymbol{q})^{2}+q^{4} D_{0}^{2}}\right. \\
& \times\left[\hat{u}_{i}^{\mathrm{R}}(\boldsymbol{q}, \omega) \hat{u}_{j}^{\mathrm{R}}(\boldsymbol{q}, \omega)+\hat{u}_{i}^{\mathrm{I}}(\boldsymbol{q}, \omega) \hat{u}_{j}^{\mathrm{I}}(\boldsymbol{q}, \omega)\right] \\
& +\left[\hat{u}_{i}^{\mathrm{R}}(\boldsymbol{q}, \omega) \hat{u}_{j}^{\mathrm{I}}(\boldsymbol{q}, \omega)-\hat{u}_{i}^{\mathrm{I}}(\boldsymbol{q}, \omega) \hat{u}_{j}^{\mathrm{R}}(\boldsymbol{q}, \omega)\right] \\
& \left.\times \frac{\omega+\boldsymbol{U} \cdot \boldsymbol{q}}{(\omega+\boldsymbol{U} \cdot \boldsymbol{q})^{2}+q^{4} D_{0}^{2}}\right\} d \boldsymbol{q} d \omega+O\left(\varepsilon^{3}\right) .
\end{aligned}
$$

From the above expression one immediately realizes that the antisymmetric part of $D_{i j}(X, T)$ is

$$
\begin{aligned}
D_{i j}^{A}(\boldsymbol{X}, T)= & \int\left[\hat{u}_{i}^{\mathrm{R}}(\boldsymbol{q}, \omega) \hat{u}_{j}^{\mathrm{I}}(\boldsymbol{q}, \omega)-\hat{u}_{i}^{\mathrm{I}}(\boldsymbol{q}, \omega) \hat{u}_{j}^{\mathrm{R}}(\boldsymbol{q}, \omega)\right] \\
& \times \frac{\omega+\boldsymbol{U} \cdot \boldsymbol{q}}{(\omega+\boldsymbol{U} \cdot \boldsymbol{q})^{2}+q^{4} D_{0}^{2}} d \boldsymbol{q} d \omega .
\end{aligned}
$$

The condition for the latter to be zero is

$$
\hat{u}_{i}^{\mathrm{R}}(\boldsymbol{q}, \omega) \hat{u}_{j}^{\mathrm{I}}(\boldsymbol{q}, \omega)-\hat{u}_{i}^{\mathrm{I}}(\boldsymbol{q}, \omega) \hat{u}_{j}^{\mathrm{R}}(\boldsymbol{q}, \omega)=0,
$$

from which sufficient conditions for its validity are immediately obtained:

$$
\hat{\boldsymbol{u}}^{\mathrm{R}}(\boldsymbol{q}, \omega)=0 \quad \forall \boldsymbol{q} \text { and } \omega \text { or } \hat{\boldsymbol{u}}^{\mathrm{I}}(\boldsymbol{q}, \omega)=0 \quad \forall \boldsymbol{q} \text { and } \omega .
$$

Conditions (48) amount to saying that if the small-scale velocities have defined parity with respect to space and time inversion, then only the symmetric part of $D_{i j}$ controls the preasymptotic scalar dynamics.

To conclude, it is worth observing that the formula (42) can be generalized to random small-scale velocity mimicking turbulent small-scale fluctuations. In this case $\hat{u}_{i}(-\boldsymbol{q},-\omega) \hat{u}_{j}(\boldsymbol{q}, \omega)$ in (42) must be replaced by $\left\langle\hat{u}_{i}(-\boldsymbol{q},-\omega) \hat{u}_{j}(\boldsymbol{q}, \omega)\right\rangle$ where the angular brackets denote the average with respect to small-scale velocity statistics. If one deals with stationary, homogeneous and isotropic fluctuations the spectral tensor $\left\langle\hat{u}_{i}(-\boldsymbol{q},-\omega) \hat{u}_{j}(\boldsymbol{q}, \omega)\right\rangle$ is invariant un$\operatorname{der} \boldsymbol{q} \rightarrow-\boldsymbol{q}$ and $\omega \rightarrow-\omega$ with the immediate consequence that

$$
\left\langle\hat{u}_{i}^{\mathrm{R}}(\boldsymbol{q}, \omega) \hat{u}_{j}^{\mathrm{I}}(\boldsymbol{q}, \omega)\right\rangle-\left\langle\hat{u}_{i}^{\mathrm{I}}(\boldsymbol{q}, \omega) \hat{u}_{j}^{\mathrm{R}}(\boldsymbol{q}, \omega)\right\rangle=0,
$$

a condition that generalizes (47).

\section{B. The case of orthogonal shears}

Although Eq. (42) is just a first-order approximation, it provides a concrete tool to estimate the eddy diffusivity, and it can be shown that for the particular case of orthogonal shears it recovers the exact solution [19]. Indeed, if the velocity field is the sum of two orthogonal shears

$$
\boldsymbol{v}(\boldsymbol{x}, t ; \boldsymbol{X}, T)=\boldsymbol{u}(\boldsymbol{x}, t)+\boldsymbol{U}(\boldsymbol{X}, T)
$$

with

$$
\boldsymbol{u}(\boldsymbol{x}, t)=(u(y, z, t), 0,0), \quad \boldsymbol{U}(\boldsymbol{X}, T)=(0, U(X, Z, T), 0)
$$

it follows from Eq. (21) that the unique nonvanishing component of the auxiliary field is the one in the direction of the small-scale velocity, and it is constant along that direction. Therefore the small-scale velocity field does not give contributions in the advective term of Eq. (21) which can exactly be solved in Fourier space to obtain

$$
\hat{\boldsymbol{\chi}}(\boldsymbol{k}, \omega ; \boldsymbol{X}, T)=\left(\frac{-\hat{\boldsymbol{u}}(\boldsymbol{k}, \omega)}{i(\omega+\boldsymbol{U} \cdot \boldsymbol{k})+k^{2} D_{0}}, 0,0\right) .
$$

\section{NUMERICAL RESULTS AND "EMPIRICAL RECIPES" FOR THE PREASYMPTOTIC TRANSPORT}

In the previous section we have discussed a perturbative solution and its possible limitations when $u / U$ and $\ell / L$ are not very small. Let us now present some numerical results and an empirical "recipe" for a constant (i.e., without space and time dependence) preasymptotic eddy diffusivity.

\section{A. Numerical results}

As an example of small-scale incompressible flows we consider a steady cellular flow [20,22,27] defined by the stream function $\psi=\psi_{0} \sin (k x) \sin (k y)$ with $\psi_{0}=u / k$ : 


$$
\boldsymbol{u}=\left(\partial_{y} \psi,-\partial_{x} \psi\right)=(u \sin (k x) \cos (k y),-u \cos (k x) \sin (k y))
$$

Its characteristic length scale is given by $\ell=2 \pi / k$ and its amplitude is $u$.

In the absence of large-scale velocity fields and for large Péclet numbers $\left(\mathrm{Pe}=u \ell / D_{0}\right)$, it is possible to show by means of simple physical arguments [28] that this periodic array of small vortices give rise to an enhancement of the effective diffusivity $D^{E} \sim D_{0} \sqrt{\mathrm{Pe}}$. A precise estimation of this constant eddy diffusivity can be obtained by the numerical solution of Eq. (21), with $\boldsymbol{U}=\mathbf{0}$.

The modifications induced on the eddy diffusivity by the presence of a large-scale flow

$$
\boldsymbol{U}=(U(X, Y, T), V(X, Y, T))
$$

with characteristic length scale $L=\ell / \epsilon$ and strength $U=u / \varepsilon$ can be estimated from Eq. (42). Thanks to the simplicity of our small-scale flow, the integral in Eq. (42) reduces to the sum of contributions of four modes $( \pm k, \pm k)$, and trivial calculations lead to

$$
\begin{aligned}
D_{i j}^{E}= & D_{0} \delta_{i j}\left\{1+\frac{1}{4} u^{2}\left[\frac{1}{(U+V)^{2}+\left(2 k D_{0}\right)^{2}}\right.\right. \\
& \left.\left.+\frac{1}{(U-V)^{2}+\left(2 k D_{0}\right)^{2}}\right]\right\}+O\left(\varepsilon^{3}\right) .
\end{aligned}
$$

For such a system the antisymmetric part $D_{i j}^{A}$ is identically zero.

Let us now focus on two idealized large-scale flows, which are representative of two broad classes of realistic situations: a steady shear

$$
\boldsymbol{U}=(U \sin (K y), 0)
$$

and a large-scale replica of the cellular flow

$$
\boldsymbol{U}=(U \sin (K x) \cos (K y),-U \cos (K x) \sin (K y)) .
$$

Their characteristic length scale is $L=2 \pi / K$ and $U$ is their amplitude. For the case of the large-scale shear, Eq. (55) reduces to

$$
D_{i j}^{E}=D_{0} \delta_{i j}\left(1+\frac{1}{2} \frac{u^{2}}{U^{2} \sin ^{2}(y)+\left(2 k D_{0}\right)^{2}}\right)+O\left(\varepsilon^{3}\right)
$$

while in the case of the large-scale cellular flow one gets

$$
\begin{aligned}
D_{i j}^{E}= & D_{0} \delta_{i j}\left\{1+\frac{1}{4} u^{2}\left[\frac{1}{U^{2} \sin ^{2}[K(x+y)]+\left(2 k D_{0}\right)^{2}}\right.\right. \\
& \left.\left.+\frac{1}{U^{2} \sin ^{2}[K(x-y)]+\left(2 k D_{0}\right)^{2}}\right]\right\}+O\left(\varepsilon^{3}\right) .
\end{aligned}
$$

In Fig. 1 we compare the exact multiple-scale solution for $D_{x x}^{E}(y)$ in the case of the large-scale shear flow with the approximation (58) and the constant estimation based on only the small-scale cellular flow, respectively. In most of the domain the first-order approximation recovers quite well the exact solution, with the exception of narrow regions where the large-scale flow vanishes and the actual diffusivity is mainly determined by the cellular flow.

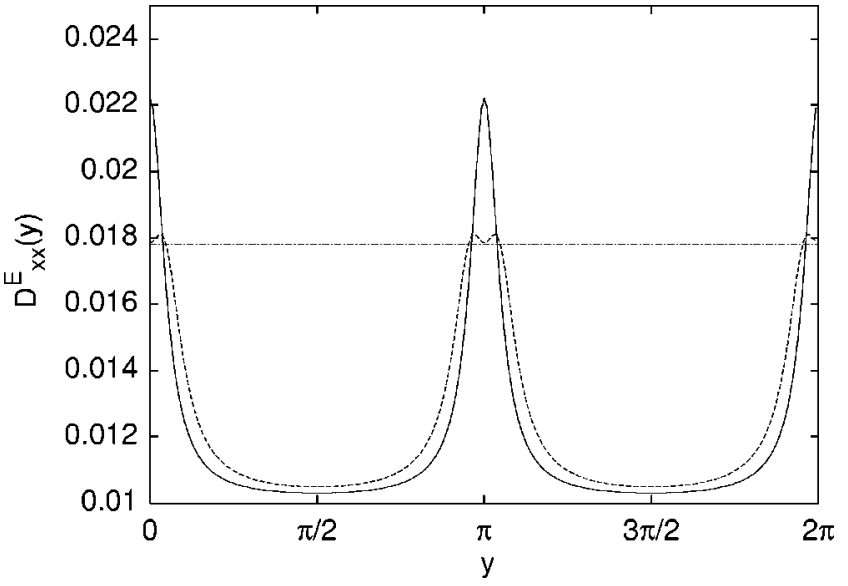

FIG. 1 . The eddy diffusivity $D_{x x}^{E}(y)$ resulting from a small-scale cellular flow superimposed on a large-scale shear in the $x$ direction. The approximation (58) (solid line) recovers quite well the exact multiple-scale solution (dashed line), except for narrow regions where the large-scale flow vanishes $(y=n \pi)$ and the actual diffusivity recovers the constant estimation based on only the small-scale cellular flow (dash-dotted line). The parameter values are $U=1, L$ $=2 \pi, u / U=1 / 4, \ell / L=1 / 8, D_{0}=0.01$. Units are made dimensionless according to Eq. (60).

In all figures and tables we show quantities made dimensionless in the form

$$
x \rightarrow \frac{x}{L_{0}}, \quad v \rightarrow \frac{v}{U_{0}}, \quad t \rightarrow t \frac{U_{0}}{L_{0}}, \quad D \rightarrow \frac{D}{L_{0} U_{0}},
$$

where $U_{0}=U$ and $L_{0}=L / 2 \pi$.

Once the first-order approximations (58) and (59) for the eddy diffusivity have been plugged into the preasymptotic large-scale equation, we compute the asymptotic eddy diffusivity at very large scales $\mathcal{L}$.

Numerical integration of the auxiliary equation (29) is advanced in time until the asymptotic eddy diffusivity given by Eq. (28) converges to its constant value. The latter is then compared with the values given by homogenization of Eq. (4) for different phase shifts between $\boldsymbol{U}$ and $\boldsymbol{u}$.

The observed variability of $D^{\mathcal{L}, e x}$ for different phase shifts provides an estimation of the accuracy of the multiple-scale results. Indeed, as already noted, the two successive homogenizations do not capture any effect induced by the phase shift.

Here, we consider the two extreme possibilities (i) the zeros of the large-scale flow coincide with the nodes of the small-scale cellular flow [case (a)]; (ii) the zeros of the largescale flow are located on the bulges of the small-scale cellular flow [case (b)].

In addition, we compute the constant eddy diffusivity $D_{i j}^{E}=\tilde{D} \delta_{i j}$ of the velocity field containing the small-scale cellular flow only. This leads to a rather crude approximation for the asymptotic eddy diffusivity $D^{\mathcal{L}, n}$ (in the following, we will refer to it as the "naive approximation").

For the large-scale shear, the asymptotic diffusion tensor $D^{\mathcal{L}}$ is diagonal and strongly anisotropic. In Fig. 2 we show its component $D_{x x}^{\mathcal{L}}$ in the direction parallel to the large-scale 


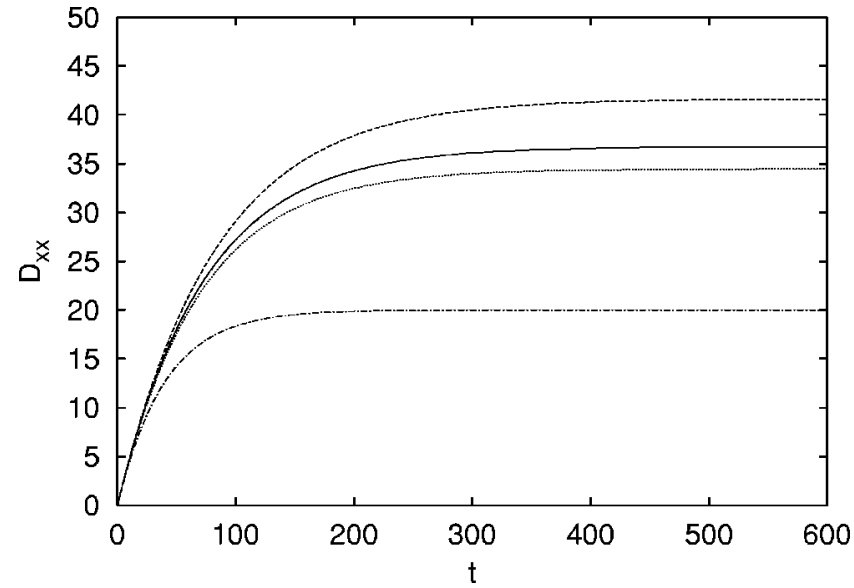

FIG. 2. Time evolution of the asymptotic eddy diffusivity in the direction of the large-scale shear up to its convergence to its constant value. The scale separation between the large-scale shear and the small-scale cellular flows is $\ell / L=1 / 4$, the ratio of amplitudes is $u / U=1 / 4$, and the molecular diffusivity is fixed to the value $D_{0}$ $=10^{-2}$. The first-order approximation in $\varepsilon=u / U$ (solid line) provides a good estimation on the actual values, which depend on the relative phase shift between the two fields: case (a) is denoted by the dashed line, case (b) is denoted by the dotted line. For comparison we also show the results obtained from the "naive estimation" (dash-dotted line) in which the effects of the large-scale flow have been neglected. Units are made dimensionless according to Eq. (60).

shear flow. The scale separation is $\ell / L=1 / 4$, the ratio of amplitudes is $u / U=1 / 4$, and the molecular diffusion is fixed to the value $D_{0}=10^{-2}$.

In the direction of the shear the effect of the small-scale flow is to reduce the asymptotic diffusion coefficient, which for the pure large-scale shear flow would be given by

$$
D_{x x}^{\mathcal{L}}=D_{0}+\frac{1}{2} \frac{U^{2}}{K^{2} D_{0}}=50.01 .
$$

Such reduction is due to interference mechanisms between small-scale and large-scale motion [29].

With our parameters the actual reduction is of the order of 20-30\%, depending on the phase shift between $\boldsymbol{U}$ and $\boldsymbol{u}$. The first-order approximation (42) for the eddy diffusivity provides a good estimation giving a reduction for $D_{x x}^{\mathcal{L}}$ of about $28 \%$ (see Table I). On the contrary the "naive approximation" gives a reduction for $D_{x x}^{\mathcal{L}}$ of about $60 \%$, which is deeply wrong.

In the transverse direction, the bare molecular diffusivity $D_{0}$ is increased by the presence of the small-scale flow. The "naive approximation" overestimates this effect, giving an enhancement of about $170 \%$ of $D_{0}$, while approximation (42) is in rather good agreement with the actual value of about $12-20 \%$.

It is worth stressing that the large errors given by the "naive approximation" rather than being consequences of finite scale separation are mainly due to the fact that the effects of the large-scale flow have been neglected in the constant eddy diffusivity $D_{i j}^{E}(\boldsymbol{X}, T)=\widetilde{D} \delta_{i j}$. Indeed, with a larger
TABLE I. Asymptotic eddy diffusivity resulting from the effects of large-scale shear flow $(U=1, L=2 \pi)$, small-scale cellular flow, and molecular diffusivity $D_{0}=0.01 . D^{\mathcal{L}, e x}$ [cases (a) and (b)] are the actual values obtained from direct homogenization of the whole velocity field $\boldsymbol{v}=\boldsymbol{U}+\boldsymbol{u} . D^{\mathcal{L}}$ and $D^{\mathcal{L}, n}$ are obtained from the homogenization of the preasymptotic equation where the preasymptotic eddy diffusivities are approximated by expression (42) and by retaining only the small-scale cellular flow, respectively. Units are made dimensionless according to Eq. (60).

\begin{tabular}{ccccc}
\hline \hline$\ell / L$ & $u / U$ & $D^{\mathcal{L}, e x}$ & $D^{\mathcal{L}}$ & $D^{\mathcal{L}, n}$ \\
\hline $1 / 4$ & $1 / 4$ & $D_{x x}=41.6^{(\mathrm{a})}, 34.5^{(\mathrm{b})}$ & 36.7 & 18.7 \\
& & $D_{y y}=0.0112^{(\mathrm{a})}, 0.0122^{(\mathrm{b})}$ & 0.0119 & 0.0267 \\
$1 / 8$ & \multirow{2}{*}{$1 / 4$} & $D_{x x}=41.5^{(\mathrm{a})}, 40.5^{(\mathrm{b})}$ & 39.6 & 28.3 \\
& & $D_{y y}=0.0112^{(\mathrm{a})}, 0.0113^{(\mathrm{b})}$ & 0.0115 & 0.0178 \\
\hline \hline
\end{tabular}

scale separation $\ell / L=1 / 8$ the approximate solution gives results within the $2 \%$ of the actual values, while the "naive approximation" still gives an error of about 30\% (see Table I).

In the case of the large-scale cellular flow (see Fig. 3) the asymptotic eddy diffusivity is isotropic, and the first-order approximation is even more robust, providing good estimations also for $\epsilon=\ell / L=1 / 4$ and $\varepsilon=u / U=1 / 2$ (see Table II). The errors of the "naive approximation" are of the order of $100 \%$.

\section{B. An empirical "recipe"}

We discuss now an empirical "recipe" to obtain a constant (i.e., having no variation in space and in time) eddy diffusivity to describe preasymptotic scales. The question is thus on whether it is possible to mimic the preasymptotic transport

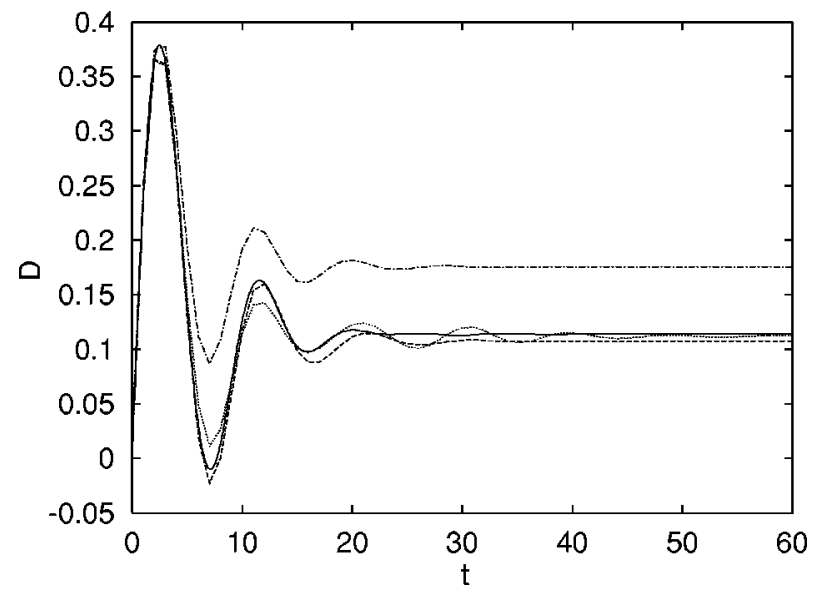

FIG. 3. The same as in Fig. 2 for the case of the large-scale cellular flow $\left(\ell / L=1 / 4, u / U=1 / 4, D_{0}=10^{-2}\right)$. Time evolution of the asymptotic eddy diffusivity $D^{\mathcal{L}}$ is well approximated by the first-order approximation (42) (solid line), while the "naive estimation" (dash-dotted) does not match the actual values which depend on the relative phase shifts between the two fields: case (a) dashed line; case (b) dotted line. Units are made dimensionless according to Eq. (60). 
TABLE II. The same as in Table I for the large-scale cellular flow.

\begin{tabular}{ccccc}
\hline \hline$\ell / L$ & $u / U$ & $U^{\mathcal{L}, e x}$ & $D^{\mathcal{L}}$ & $D^{\mathcal{L}, n}$ \\
\hline $1 / 4$ & $1 / 2$ & $0.111^{(\mathrm{a})}, 0.123^{(\mathrm{b})}$ & 0.135 & 0.209 \\
$1 / 4$ & $1 / 4$ & $0.107^{(\mathrm{a})}, 0.112^{(\mathrm{b})}$ & 0.113 & 0.175 \\
\hline \hline
\end{tabular}

by means of an average diffusion tensor $D_{i j}^{E, a}$, which still takes into account the effects of the large-scale flow $\boldsymbol{U}$ but does not depend on the position. In general it is not clear which is the correct way for averaging $D_{i j}(\boldsymbol{X}, T)$ to obtain a constant, but still anisotropic diffusion tensor. Here, we propose a possible "recipe" which is inspired by the multiplescale approach. The idea consists in applying the homogenization technique just on the diffusive term of the preasymptotic equation, obtaining $D_{i j}^{E, a}$ in the same way as for $D^{\mathcal{L}}$ :

$$
D_{i j}^{E, a}=\frac{\left\langle D_{i k} \partial_{k} \chi_{j}\right\rangle+\left\langle D_{j k} \partial_{k} \chi_{i}\right\rangle}{2}+\frac{\left\langle D_{i j}\right\rangle+\left\langle D_{j i}\right\rangle}{2},
$$

where the vector field $\boldsymbol{\chi}$ is a solution of the auxiliary equation

$$
\partial_{t} \chi_{k}+(\boldsymbol{U} \cdot \boldsymbol{\partial}) \chi_{k}-\partial\left(D_{i j} \partial_{j} \chi_{k}\right)=\partial_{i} D_{i k} .
$$

Although the recipe (62), (63) cannot be rigorously proved, it is possible to give a rough argument in favor of it. Equations (62) and (63) can be seen as the analogs of Eqs. (28) and (29) in which only the eddy-diffusivity contributions to the asymptotic diffusion tensor have been retained.

The above discussed preaveraged constant diffusion tensor is potentially interesting in applications, where it is almost impossible to deal with space-dependent eddy diffusivities. Let us stress the fact that $D_{i j}^{E, a}$ in Eq. (62) is constant, but it takes into account the effects of the large-scale flow to provide a correct estimation of an effective diffusion tensor.

Numerical simulations of the preasymptotic equation (23) in which $D_{i j}(\boldsymbol{X}, T)$ is replaced by the constant tensor $D_{i j}^{E, a}$ confirms that this averaging recipe leads to considerable improvements with respect to the "naive approximation" obtained without considering the effects of the large-scale flow.

Table III shows the results in the case of the large-scale shear flow, where this averaging leads to a rather good approximation $D^{\mathcal{L}, a}$ for the asymptotic eddy diffusivity; similar results hold for the case of large-scale cellular flow.

TABLE III. The same as in Table I. The asymptotic eddy diffusivity $D^{\mathcal{L}, a}$ is obtained from the homogenization of the preasymptotic equation where the preasymptotic eddy diffusivity is approximated by the constant value given by Eq. (62).

\begin{tabular}{ccccc}
\hline \hline$\ell / L$ & $u / U$ & $D^{\mathcal{L}, e x}$ & $D^{\mathcal{L}, a}$ & $D^{\mathcal{L}, n}$ \\
\hline $1 / 4$ & $1 / 4$ & $D_{x x}=41.6^{(\mathrm{a})}, 34.5^{(\mathrm{b})}$ & 42.1 & 18.7 \\
& & $D_{y y}=0.0112^{(\mathrm{a})}, 0.0122^{(\mathrm{b})}$ & 0.0118 & 0.0267 \\
$1 / 8$ & \multirow{2}{*}{$1 / 4$} & $D_{x x}=41.5^{(\mathrm{a})}, 40.5^{(\mathrm{b})}$ & 43.6 & 28.3 \\
& & $D_{y y}=0.0112^{(\mathrm{a})}, 0.0113^{(\mathrm{b})}$ & 0.0115 & 0.0178 \\
\hline \hline
\end{tabular}

\section{MULTIPLE-SCALE EXPANSION AND RENORMALIZATION GROUP}

In previous sections we studied the problem of large-scale transport in field varying on two separated scales that we called large and small scales, respectively.

In practical applications, one has to deal with advecting velocity fields having almost a continuum of active scales. In this latter case, we can write

$$
\boldsymbol{u}(\boldsymbol{x}, t)=\sum_{n=0}^{N} \boldsymbol{u}_{n}(\boldsymbol{x}, t)=\boldsymbol{u}_{0}(\boldsymbol{x}, t)+\delta \boldsymbol{u}(\boldsymbol{x}, t)
$$

where the Fourier transform of $\boldsymbol{u}_{n}(\boldsymbol{x}, t)$ is picked on wave numbers around $k_{n} \sim l_{n}^{-1}=2^{-n} l_{0}^{-1}$. Denoting with $E(k)$ the energy spectrum, one has

$$
\frac{1}{2}\left\langle\left|\boldsymbol{u}_{n}(\boldsymbol{x}, t)\right|^{2}\right\rangle \simeq \int_{k_{n}}^{k_{n+1}} E(k) d k .
$$

We are now ready to address the following question: What is the effect of $\delta \boldsymbol{u}(\boldsymbol{x}, t)$ on the effective asymptotic eddy diffusivity? In other words, we aim at obtaining an effective large-scale equation and determining the dependence of $\boldsymbol{U}^{E}$ and $D^{E}$ on $\delta \boldsymbol{u}(\boldsymbol{x}, t)$ and $D_{0}$, respectively.

A natural way to answer our question is to exploit the renormalization group point of view. The basic idea proceeds through these steps.

(1) Starting from the original equation (4), one considers the field

$$
\boldsymbol{U}_{N-1}(\boldsymbol{x}, t)=\sum_{n=0}^{N-1} \boldsymbol{u}_{n}(\boldsymbol{x}, t)
$$

as the one at large scales and $\boldsymbol{u}_{N}(\boldsymbol{x}, t)$ as the contribution at small scales. Recalling the results of the multiple-scale expansion reported in Sec. II, we can write the effective equation for the field including the contribution up to the scale $N-1$, i.e.,

$$
\partial_{t} \theta+\boldsymbol{U}_{N-1}^{E} \cdot \nabla \theta=\nabla\left(D_{N-1}^{E} \nabla \theta\right),
$$

where $\boldsymbol{U}_{N-1}^{E}$ and $D_{N-1}^{E}$ are determined by the multiple-scale analysis of Secs. II and III. It is rather obvious that it is almost impossible to repeat in full detail the multiple-scale procedure. On the other hand, if one is interested only in the order of magnitude, interesting results can be obtained by neglecting the dependence on $\boldsymbol{x}$. In this spirit we obtain

$$
D_{N-1}^{E} \simeq D_{0}+\text { const } \frac{D_{0}\left\langle\left|\boldsymbol{u}_{N}\right|^{2}\right\rangle k_{N}^{2}}{\left(D_{0} k_{N}^{2}\right)^{2}+\left(k_{N}\left|\boldsymbol{U}_{N-1}\right|\right)^{2}}
$$

and

$$
\boldsymbol{U}_{N-1}^{E}=\boldsymbol{U}_{N-1}(\boldsymbol{x}, t)+\delta \boldsymbol{U}_{N-1}
$$

where $\delta \boldsymbol{U}_{N-1}$ is the compressible contribution originating from the dependence of $D_{N-1}^{E}$ on $\boldsymbol{x}$.

(2) As a second step, one now has to iterate the previous procedure. In order to simplify the computation, as before we do not take into account either the dependence of $D_{N-1}^{E}$ on $\boldsymbol{x}$ or the compressible correction on $\boldsymbol{U}_{N-1}^{E}$. We have just to re- 
place $D_{0}$ with $D_{N-1}^{E}, \boldsymbol{U}_{N-1}$ with $\boldsymbol{U}_{N-2}, k_{N}$ with $k_{N-1}$, and so on. When doing so, we arrive at

$$
D_{N-2}^{E} \simeq D_{N-1}^{E}+\mathrm{const} \frac{D_{N-1}^{E}\left\langle\left|\boldsymbol{u}_{N-1}\right|^{2}\right\rangle k_{N-1}^{2}}{\left(D_{N-1}^{E} k_{N-1}^{2}\right)^{2}+\left(k_{N-1}\left|\boldsymbol{U}_{N-1}\right|\right)^{2}}
$$

and similarly for $\boldsymbol{U}_{N-2}^{E}$, and so on, for $N-3, N-4$, etc.

The effective asymptotic eddy diffusivity $D^{E}$ is obtained by iterating the recursive relation (70). Two interesting limits have been identified.

(i) The dominant term in the denominator of Eq. (70) is $\left(D_{N-1}^{E} k_{N-1}^{2}\right)^{2}$ and the recursive formula becomes

$$
D_{N-2}^{E} \simeq D_{N-1}^{E}+\mathrm{const} \frac{\left\langle\left|\boldsymbol{u}_{N-1}\right|^{2}\right\rangle}{D_{N-1}^{E} k_{N-1}^{2}} .
$$

(ii) The dominant term in the denominator of Eq. (70) is $\left(k_{N-1}\left|\boldsymbol{U}_{N-1}\right|\right)^{2}$ and we thus have

$$
D_{N-2}^{E} \simeq D_{N-1}^{E}\left(1+\mathrm{const} \frac{\left\langle\left|\boldsymbol{u}_{N-1}\right|^{2}\right\rangle}{\left|\boldsymbol{U}_{N-1}\right|^{2}}\right)
$$

The relation (71) coincides with the result obtained by Moffatt [30]. Iterating (71) one easily obtains

$$
D^{E} \sim \sqrt{\int k^{-2} E(k) d k}
$$

i.e., an eddy diffusivity which does not depend on the molecular diffusivity $D_{0}$.

On the contrary, exploiting the fact that $\boldsymbol{U}_{N_{1}} \simeq \boldsymbol{u}_{0}$ from (72) one has

$$
D^{E} \sim D_{0}\left(1+\text { const } \sum_{n} \frac{\left\langle\left|\boldsymbol{u}_{n}\right|^{2}\right\rangle}{\left|\boldsymbol{u}_{0}\right|^{2}}\right) \propto D_{0} .
$$

In summary, from the iteration of the recursive rule (70) one can obtain at least two fixed points. In the first case the asymptotic eddy diffusivity is determined only from the velocity field and it does not depend on $D_{0}$. This allows for values of $D^{E}$ much larger than $D_{0}$. In the second limit, one has a small variation of the asymptotic eddy diffusivity which remains of the same order as $D_{0}$.

\section{CONCLUSIONS}

We have investigated both analytically and numerically the preasymptotic transport of a passive scalar field on large scales, say, of order $L$. The velocity field advecting the scalar is formed by a large-scale component $\boldsymbol{U}$ varying on scales of order of $L$ and by a small-scale fluctuation $\boldsymbol{u}$, which varies on scales of order of $\ell$ much smaller than $L$. The presence of a small parameter $\ell / L$ naturally allows a perturbative analysis: the so-called multiple-scale strategy.

The following results must be emphasized.

(1) Preasymptotic scalar transport is ruled by a FokkerPlanck equation involving an effective eddy-diffusivity field and an effective advecting velocity. Although explicit expressions for such effective fields cannot be determined in general, nevertheless it is apparent that the eddy diffusivity does depend on the large-scale advecting velocity. This is in contrast with the usual point of view which sees the eddy diffusivity as the cumulative result of interactions involving only the small scales. This aspect can be rather relevant in a geophysical context [31].

(2) If one makes the additional assumption that smallscale fluctuations are sufficiently weaker than the large-scale fluctuations (i.e., $u / U \ll 1$ ), an approximate explicit expression for the eddy-diffusivity tensorial field can be obtained. This expression makes explicit the dependence of the eddy diffusivity on the large-scale velocity, which, in turn, carries a spatiotemporal dependence on large scales.

(3) If the small-scale velocity $\boldsymbol{u}$ has defined parity under spatial and temporal inversion, only the symmetric part of $D_{i j}$ is relevant for the preasymptotic dynamics. The same conclusion holds if $\boldsymbol{u}$ is a small-scale stationary, homogeneous, and isotropic turbulent field.

(4) We have tested numerically the validity of our approximated expression for the eddy diffusivity for values of $u / U$ and $\ell / L$ not necessarily much less than unity. As expected, the range of reliability of our approximation extends to finite values of the above ratios. This seems an important conclusion for applications in the realm of geophysics and oceanography.

(5) Exploiting the explicit formula for the eddy diffusivity, we have presented a generalization of our results to situations with a continuum of active scales. This procedure gives rise to a sort of renormalization group through which it is possible to extract two completely different regimes of transport.

We would like to conclude with a short discussion on the applicability of our results and, more generally, of multiplescale techniques to geophysical problems. As far as the first point is concerned, a paradigmatic example of a possible application is provided by the investigation of pollutant dispersion in the planetary boundary layer. The latter is a thin $(\sim 1000 \mathrm{~m})$ atmospheric layer near the ground, where the airflow is strongly driven by sink and source forcing terms arising from the bottom boundary, e.g., due to the orography. The decomposition of the velocity field as $\boldsymbol{v}=\boldsymbol{u}+\boldsymbol{U}, \boldsymbol{u}$ being a fluctuating random component, whose statistical properties are prescribed and $\boldsymbol{U}$ a slowly varying part, is a standard decomposition. By way of example, the slow component $\boldsymbol{U}$ describes synoptic variations while the fast component $\boldsymbol{u}$ modelizes, for instance, orographic excitations.

Let us now point out some important limitations in the applicability of the multiple-scale analysis to geophysical problems. A first obvious limit comes from the separation between the characteristic scales of the flow. The multiplescale approach is strictly valid only in the case of large separation, while the typical separation of scales and amplitudes in realistic geophysical flows is not very large. Actually, this does not seem a severe restriction, since the results obtained in the limit of infinite separation provide rather good approximations also valid for moderate separations (see, e.g., the numerical results of Sec. IV).

Moreover, the multiple-scale approach requires a detailed 
knowledge of the Eulerian velocity field, which is not always available experimentally. It thus seems to us that an attempt to build a preasymptotic equation for the transport, using only Lagrangian experimental data, should be a further important step toward a satisfactory understanding of how to modelize large-scale transport in geophysical flows.

\section{ACKNOWLEDGMENTS}

This work was supported by Cofin "Sistemi Complessi e Problemi a Molti Corpi." Numerical simulations have been performed at CINECA (INFM parallel computing initiative).
[1] W. E and B. Engquist, Not. Am. Math. Soc. 50, 1062 (2003).

[2] L. M. Gierasch and J. King, Protein Folding (American Association for the Advancement of Science, Washington, D.C., 1990).

[3] Stochastic Climate Models, edited by P. Imkeller and J.-S. von Storch (Birkhäuser, Boston, 2001).

[4] H. Mori, Prog. Theor. Phys. 33, 423 (1965).

[5] R. Zwanzig, J. Stat. Phys. 9, 215 (1973).

[6] D. Wirosoetisno and T. G. Shepherd, Physica D 141, 141 (2000).

[7] W. Just, K. Gelfert, N. Baba, A. Riegert, and H. Kantz, J. Stat. Phys. 112, 277 (2003).

[8] K. Hasselmann, Tellus 28, 473 (1976).

[9] A. Majda, I. Timofeyev, and E. Vanden Eijnden, Proc. Natl. Acad. Sci. U.S.A. 96, 14687 (1999).

[10] A. Einstein, Ann. Phys. 17, 549 (1905).

[11] P. Langevin, C. R. Hebd. Seances Acad. Sci. 146, 530 (1908).

[12] A. Wentzel and M. Freidlin, Random Perturbations of Dynamical Systems (Springer-Verlag, New York, 1987).

[13] M. Cassandro and G. Jona-Lasinio, Adv. Phys. 27, 913 (1978).

[14] T. G. Kurtz, J. Funct. Anal. 12, 55 (1973).

[15] G. C. Papanicolaou, Rocky Mt. J. Math. 6, 653 (1976).

[16] D. Givon, R. Kupferman, and A. Stuart, Nonlinearity 17, R55 (2004).

[17] E. Vanden-Eijnden, Commun. Math. Sci. 1, 385 (2003).
[18] I. Fatkullin and E. Vanden-Eijnden, J. Comput. Phys. 200, 605 (2004).

[19] A. Mazzino, Phys. Rev. E 56, 5500 (1997).

[20] L. Biferale, A. Crisanti, M. Vergassola, and A. Vulpiani, Phys. Fluids 7, 2725 (1995).

[21] A. Bensoussan, J.-L. Lions, and G. Papanicolaou, Asymptotic Analysis for Periodic Structures (North-Holland, Amsterdam, 1978).

[22] A. J. Majda and P. R. Kramer, Phys. Rep. 314, 234 (1999).

[23] D. Mc Laughlin, G. C. Papanicolaou, and O. Pironneau, SIAM (Soc. Ind. Appl. Math.) J. Appl. Math. 45, 780 (1985).

[24] G. A. Pavliotis and P. R. Kramer, in Proceedings of the Fourth International Conference on Dynamical Systems and Differential Equations, Wilmington, NC, 2002 (unpublished), pp. 1-8.

[25] R. Mauri, Phys. Rev. E 68, 066306 (2003).

[26] M. Vergassola and M. Avellaneda, Physica D 106, 148 (1997).

[27] T. H. Solomon and J. P. Gollub, Phys. Rev. A 38, 6280 (1988).

[28] B. Y. Pomeau, C. R. Acad. Sci., Ser. II: Mec., Phys., Chim., Sci. Terre Univers 301, 1323 (1985).

[29] A. Mazzino and M. Vergassola, Europhys. Lett. 37, 535 (1997).

[30] H. K. Moffatt, J. Fluid Mech. 106, 27 (1981).

[31] M. Cencini, A. Mazzino, S. Musacchio, R. Pasmanter, and A. Vulpiani (unpublished). 